\title{
Dynamic Change of Focal Fatty Sparing in Non- Alcoholic Fatty Liver Disease after Treatment with Sitagliptin
}

\section{Introduction \\ $\nabla$}

Non-alcoholic fatty liver disease (NAFLD) is considered to be a hepatic manifestation of metabolic syndrome and is associated with insulin resistance, type 2 diabetes mellitus, obesity, hypertension, and dyslipidemia (T. Fukuhara et al. Hepatogastroenterology 2014; 61: 323-328).

The current gold standard for the diagnosis of hepatic steatosis is a liver biopsy with a histopathological evaluation. However, less invasive and highly accurate diagnostic methods for the detection of fatty liver are currently being advocated, including abdominal ultrasound, CT, and MRI. Abdominal ultrasound may be the best and most available diagnostic modality for an initial fatty liver diagnosis (O.W. Hamer et al. Radiographics 2006; 26: 1637-1653).

Based on diagnostic images, there are 3 established fatty liver patterns (O.W. Hamer et al. Radiographics 2006; 26: 1637-1653): i/ diffuse homogeneous fatty liver, ii/ diffuse fatty liver with focal fatty sparing (i.e., with areas without steatosis), and iii/ focal fatty lesions in an otherwise normal liver.

The dipeptidyl peptidase-4 (DPP-4) inhibitor, sitagliptin, has been reported to improve or even reverse hepatic steatosis in patients with type 2 diabetes mellitus (T. Fukuhara et al. Hepatogastroenterology 2014; 61: 323-328).

\section{Case Description \\ $\nabla$}

A 49-year-old woman with a history of obesity, a 2-year history of metformintreated type 2 diabetes mellitus and hyperlipoproteinemia, and a 7-year history of arterial hypertension was evaluated in our diabetes outpatient clinic. Her medication included daily metformin $3000 \mathrm{mg}$ (antidiabetic drug), celiprolol $200 \mathrm{mg}$ (beta blocker), ramipril $5 \mathrm{mg}$ (angiotensin converting enzyme inhibitor), amiloride

\section{License terms}

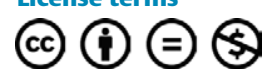

$5 \mathrm{mg}$ (diuretic) and hydrochlorothiazide $50 \mathrm{mg}$ (diuretic). The patient had no history of alcohol intake and no history of smoking.

Starting with her initial diagnosis of type 2 diabetes mellitus, her regular annual abdominal ultrasonographic examination, performed by the same sonologist, revealed severe diffuse homogeneous hepatic steatosis (A.J. Sanyal. Gastroenterology 2002; 123: 1705-1725). The patient had no history or presence of other hepatic disease, such as alcoholic fatty liver disease, drug-induced liver disease, chronic viral hepatitis B or C, autoimmune hepatitis, primary biliary cirrhosis, sclerosing cholangitis, Wilson's disease, hemochromatosis, $\alpha$-1-antitrypsin deficiency. Liver blood tests were repeatedly in the normal range. With this clinical setting the diagnosis of non-alcoholic fatty liver disease was very probable.

In May 2012, because of poor diabetes compensation, $100 \mathrm{mg}$ daily of sitagliptin (DPP-4 inhibitor) was added to her abovementioned medications. In April 2013 (eleven months after initiation of sitagliptin), a routine annual abdominal ultrasound examination showed in liver segment 2 a hypoechoic geographically configured $7.55 \mathrm{~cm}$ large lesion with poorly delineated margins and with no spaceoccupying effect in B-mode ( $\bullet$ Fig. 1). Normal vascularization was detected using a color Doppler ultrasound examination. This finding was very suspicious for focal fatty sparing (FFS), i. e., decreased fat content in specific liver areas. The diagnosis of FFS was confirmed using magnetic resonance imaging, since the patient refused liver biopsy.

15 months after the start of sitagliptin treatment, the patient refused continued treatment with sitagliptin. Surprisingly, 16 months after the discontinuation of sitagliptin, a reduction in the maximum diameter (from $7.55 \mathrm{~cm}$ to $3.51 \mathrm{~cm}$ ) of FFS was detected ( $\bullet$ Fig. 2). All sonographic evaluations were performed by the same very experienced sonologist, using a Phillips HD XE 11 ultrasound system with a 2-5 $\mathrm{MHz}$ convex transducer.
The patient continued to have very poor dietetic compliance and her lifestyle was hypokinetic. During the described period (between May 2012 and January 2015), she experienced a weight gain from 82 to $84.9 \mathrm{~kg}$, her BMI rose from 34.1 to $35.3 \mathrm{~kg} /$ $\mathrm{m}^{2}$, her HbA1c rose from 46 to $59 \mathrm{mmol} /$ mol, and her homeostatic model assessment-insulin resistance (HOMA-IR) score increased from 1.24 to $6.39 \mathrm{mU} \cdot \mathrm{mmol} / \mathrm{l}^{2}$. Liver tests were in the normal range during the treatment with sitagliptin as well as later on.

\section{Discussion \\ $\nabla$}

FFS is a frequently encountered condition, which is typically located in liver segments 2, 4, and 5 (i.e., adjacent to the ligamentum falciforme or ligamentum venosum, in the gallbladder fossa, and in the porta hepatis). The mechanisms of development of focal fatty sparing in the steatotic liver have been reported to be related to aberrant venous circulation (especially an aberrant course of the left gastric vein or gallbladder veins), arterioportal shunts, and decreased portal per-

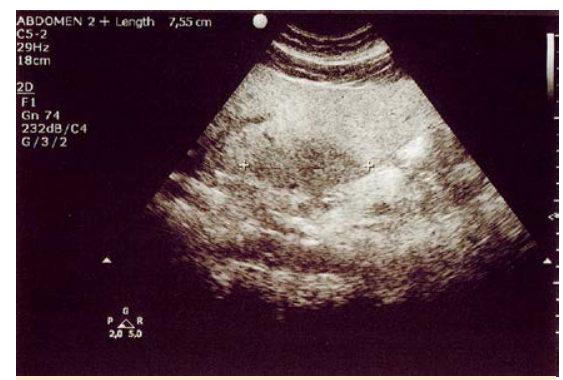

Fig. 1 Transabdominal US in B-mode examination shows in liver segment 2 hypoechoic geographically configured lesion (between crosses) with poorly delineated margins, and with no space-occupying effect. The maximal diameter of the lesion is $7.55 \mathrm{~cm}$.

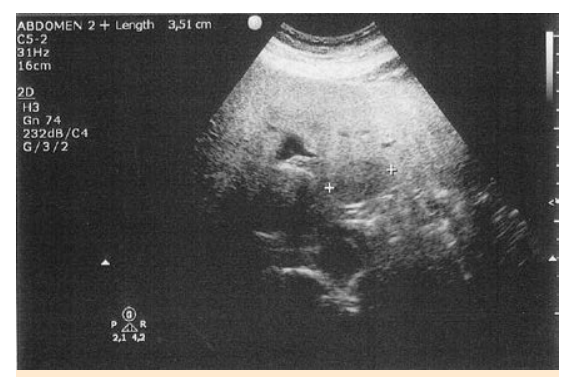

Fig. 2 Transabdominal US in B-mode examination shows in liver segment 2 the same lesion (between crosses) with a reduction in the maximum diameter to $3.51 \mathrm{~cm}$. 
fusion in the above-mentioned liver segments, which leads to localized dilution of nutrient levels in the blood flowing into the FFS (L. Liu et al. J Ultrasound Med 2008; 27: 387-394).

In our patient, we found new FFS in previously diffuse homogeneous fatty liver after sitagliptin administration. We hypothesize that this finding results from "demasking" of FFS in diffuse homogeneous hepatic steatosis in an individual likely to have aberrant venous liver circulation. Since treatment with sitagliptin is known to decrease fat content in hepatocytes and that the effect can occur rapidly in areas with diminished portal perfusion, i.e., with diminished nutrients supplies from the intestines.

Wu et al. (S. Wu et al. J Ultrasound Med 2013; 32: 1695-1702) documented that FFS is frequently encountered in non-alcoholic hepatic steatosis (approximately in $19.2 \%$ ) and that FFS may change with fatty liver changes over time. The same authors (S. Wu et al. J Ultrasound Med 2014; 33: 1447-1452), using abdominal ultrasound examinations, followed 301 patients with diffuse homogeneous NAFLD over 3 years and found no new FFS. However, this study had an observational design in which no diet, exercise, weight reduction, or pharmacological interventions had been implemented.

The observations made in our patient were found after pharmacological intervention. As such, treatment with sitagliptin is the only reasonable explanation for the focal decline of steatosis in our patient. Other influences on focal regression of hepatic steatosis, such as weight reduction, physical exercise, better diabetes compensation, or improvement in insulin resistance, could be fully excluded in our patient. Moreover, we observed a reduction in the maximum diameter of FFS 16 months after discontinuation of sitagliptin. This observation further supports our hypothesis.
Growing evidence suggests possible efficacy of incretin-based therapies, especially of (DPP-4) inhibitors in the reduction of hepatic steatosis. To our knowledge, this is the first report that an FFS may appear in the clinical course of NAFLD, especially after treatment with sitagliptin. Such findings could be challenging for ultrasonographic interpretation. However, with widespread use of DPP-4 inhibitors, this challenge will need to be met.

\section{Acknowledgement \\ $\nabla$}

The paper was supported by project NT12407-4/2011 of Internal Grant Agency of the Ministry of Health of the Czech Republic.

\footnotetext{
I. Hoffmanová, P. Škrha, A. Šedo, M. Anděl; Czech Republic
} 